\title{
PERCEPÇÃO DOS INDIVÍDUOS SOBRE O ABANDONO DO APARELHO DE
}

\author{
AMPLIFICAÇÃO SONORA INDIVIDUAL
}

\author{
Erasmo Daniel Ferreira ${ }^{1}$ \\ Luiza Augusta Rosa Rossi-Barbosa² \\ Lucilia Jezini Meira ${ }^{3}$ \\ Mirna Rossi Barbosa ${ }^{4}$ \\ Helena Jezini Meira Caldeira ${ }^{5}$ \\ Cristina Andrade Sampaio ${ }^{6}$ \\ Recebido em: 18 abr. 2018 \\ Aceito em: 23 abr. 2019
}

RESUMO: Objetivo: Este estudo buscou compreender a percepção dos indivíduos com problemas auditivos sobre 0 abandono do aparelho de amplificação sonora individual (AASI) conhecendo as situações vividas antes e após seu uso. Métodos: Foram realizadas entrevistas semiestruturadas com abordagem fenomenológica com dez indivíduos que participaram de estudo anterior e relataram terem abandonado o uso do AASI. A análise das falas seguiu os passos de descrição, redução e interpretação fenomenológica. Resultados: Pode-se observar que os familiares são os principais incentivadores para utilização do AASI. Alguns indivíduos relataram querer ouvir algo específico, ou seja, uma situação que mais gostaria de ouvir. As principais queixas para o abandono do aparelho auditivo foram o barulho, o incômodo e a estética. O motivo do não retorno se deveu ao fato de depender de outros e esquecimento. Conclusão: A percepção da aceitação do aparelho deverá ser avaliada durante o aconselhamento e retornos ao serviço. A assistência domiciliar aos usuários de aparelho de amplificação sonora individual poderá facilitar o uso deste.

Palavras-chave: Auxiliares de audição; Fonoaudiologia; Perda Auditiva; Percepção Auditiva; Pesquisa Qualitativa.

\section{PERCEPTION OF INDIVIDUALS ABOUT THE ABANDONMENT OF THE HEARING AID}

ABSTRACT: Purpose: This study sought to understand the perception of individual's with hearing problems regarding the abandonment of hearing aids by coming to know the lived experiences before and after its use. Methods: Semi-structured interviews with ten individuals were conducted with a phenomenological approach. Results: It was observed that family-members were the primary motivators for use of the hearing aid. Some people explained that they wanted to hear something specific for example a situation that they wanted to hear. The main complaints for abandoning the hearing aid was the noise, discomfort and esthetic concerns. He motive for not taking up use of the aid again is blamed on others or to forgetting. Conclusion: The perception of the acceptance of the device should be evaluated during consultations and in follow-ups. Domestic health visits to users of the device could facilitate its use.

\footnotetext{
${ }^{1}$ Faculdades Unidas do Norte de Minas - MG.

2 Universidade Estadual de Montes Claros - MG.

${ }^{3}$ Faculdades Unidas do Norte de Minas - MG.

${ }^{4}$ Faculdades Unidas do Norte de Minas - MG.

${ }^{5}$ Faculdades Unidas do Norte de Minas - MG.

6 Universidade Estadual de Montes Claros - MG.
} 
Keywords: Hearing Aids; Speech; Language and Hearing Sciences; Hearing Loss; Auditory Perception.

\section{INTRODUÇÃO}

A audição é fundamental para a comunicação humana. A incapacidade de ouvir pode gerar danos no convívio social, profissional e psicológico (MONDELLI, MAGALHÃES, LAURIS, 2011). Programas de saúde pública foram criados para diagnosticar e reabilitar indivíduos com declínio da audição possibilitando o desenvolvimento das habilidades sociais, linguísticas e cognitivas (DIDONÉ et al., 2014). A Política Nacional de Atenção à Saúde Auditiva (PNASA) foi instituída em 2004, buscando desenvolver uma rede hierarquizada e integrada entre a atenção básica, média e alta complexidade, que garanta a promoção de saúde, diagnóstico, e reabilitação auditiva, englobando a adaptação de aparelho de amplificação sonora individual (AASI), a terapia fonoaudiológica e o acompanhamento, aos indivíduos com perdas auditivas em diferentes idades (BRASIL, 2004a; MONDELLI, MAGALHÃES, LAURIS, 2011).

Pacientes com problema de audição entram nos serviços de saúde auditiva para melhorar sua função auditiva e consequentemente seu desempenho comunicativo. Com a implementação da PNASA, tornou-se necessário avaliar os efeitos do uso do AASI, a fim de promover um tratamento eficaz (COOK, HAWKINS, 2007). Desta forma, foram criados instrumentos de avaliação de benefício e satisfação do paciente (BRASIL, 2004b). Todavia, os instrumentos de avaliação da satisfação são bastante objetivos e nem sempre permitem a compreensão do que o usuário pensa, quer e planeja, não analisando amplamente as condições de vida na busca da sua qualidade (PENTEADO, SERVILHA, 2004).

Estudos registraram um número expressivo de indivíduos que abandonaram ou apresentaram uso insatisfatório do AASI (BARBOSA et al., 2013; BARBOSA, et al., 2015). Para conhecer os motivos do abandono do aparelho pelos usuários, é necessário compreender sua maneira de agir, sentir e pensar. Desta forma, a satisfação deve ser avaliada levando em consideração que a sua construção depende exclusivamente de impressões subjetivas de um paciente (HOSFORD-DUNN, HUSH, 2000; MONDELLI, MAGALHÃES, LAURIS, 2011). O objetivo deste estudo foi procurar compreender a percepção dos indivíduos com problemas auditivos sobre o abandono do AASI conhecendo as situações vividas antes e após o uso do aparelho.

\section{MATERIAL E MÉTODOS}

Utilizou-se a metodologia qualitativa por possibilitar a interpretação dos resultados em sua totalidade buscando a percepção de algum fenômeno no contexto, uma vez que pretende "[...] captar não só a aparência do fenômeno, como também sua essência" (TRIVIÑOS, 1994). A fenomenologia é um recurso apropriado para pesquisar o modo como 
a experiência é vivida. A investigação fenomenológica trabalha com aquilo que faz sentido para o sujeito, o que representa a possibilidade de ampliar a compreensão em relação ao fenômeno indagado, permitindo mostrar, descrever e compreender os fenômenos vividos (ZANARDI, YAMADA, BEVILACQUA, 2009).

Ao levar em consideração o objetivo do estudo e o perfil dos participantes, optouse pela entrevista semiestruturada, que parte da elaboração de um roteiro. Sua qualidade consiste em enumerar de forma mais abrangente possível as questões que o pesquisador necessita abordar em campo, a partir de hipóteses ou pressupostos advindos da definição do objeto de estudo (MINAYO, 2006).

A seleção dos entrevistados foi feita a partir de uma lista de 74 indivíduos contemplados com AASI entre 2010 e 2011, que informaram terem abandonado o uso do aparelho em estudo (quantitativo) anterior (BARBOSA et al., 2013). Desta lista, organizada em ordem alfabética, foram escolhidos, de cinco em cinco, quinze indivíduos. Porém, o número de entrevistados seguiu o critério de saturação do conteúdo das falas, sendo coletados dez depoimentos.

Os pesquisadores estiveram nas residências dos entrevistados e as falas foram gravadas após o conhecimento do estudo e assinatura do Termo de Consentimento Livre e Esclarecido. Os entrevistadores puderam intervir em eventuais questionamentos para esclarecer dúvidas sobre o tema e/ou para compreender determinadas respostas. Foi dada atenção não somente à fala, mas também à comunicação não verbal, porque processos interativos envolvem diferentes aspectos.

O primeiro passo do método fenomenológico constou da descrição, momento de apreensão dos significados que expressa a experiência consciente do sujeito. O segundo passo foi a redução fenomenológica, que consistiu na crítica reflexiva dos conteúdos da descrição, verificando as partes essenciais, identificando os pontos significativos. $O$ terceiro passo referiu-se à interpretação fenomenológica, compreendendo dentro da perspectiva do pesquisador (MARTINS, 1992).

Para identificar os participantes, utilizou-se a letra $E$ (Entrevistado), o número de ordem da entrevista seguido da letra $\mathrm{M}$ para masculino ou $\mathrm{F}$ para feminino, e a idade. $\mathrm{A}$ coleta de dados realizou-se conforme os aspectos éticos e legais, segundo a Resolução no 466 de 12 de dezembro de 2012 do Conselho Nacional de Saúde que regulamenta pesquisas com seres humanos. A pesquisa foi aprovada pelo Comitê de Ética da Universidade Estadual de Montes Claros sob o parecer 3042.

\section{RESULTADOS E DISCUSSÃo}

No primeiro momento foi necessário verificar aqueles sujeitos que não estavam fazendo uso do aparelho auditivo, moradores na cidade de Montes Claros e, assim, por meio das referidas entrevistas, iniciar a apreensão dos significados por eles atribuídos a esse abandono. Dos dez entrevistados seis eram do gênero masculino, a média de idade 
foi 79,6 anos, oito deles com o fundamental incompleto, nove aposentados. Nove foram adaptados bilateralmente e quanto ao tipo de aparelho recebido, sete foram do tipo BTE, dois ITC e um CIC.

A redução fenomenológica permitiu, ao transcrever seus discursos, buscar similaridades, divergências e convergências e traçar paralelos das situações vividas antes e após o uso do aparelho auditivo, surgindo assim, temas que englobam um significado para uma interpretação fenomenológica. Os temas, discutidos e apresentados com os recortes das falas dos sujeitos serão apresentados a seguir.

\section{DE QUEM FOI A VONTADE DE COLOCAR O APARELHO AUDITIVO}

Pode-se perceber que a família dos usuários entrevistados no presente estudo é a principal incentivadora para a utilização do AASI.

\footnotetext{
"Genro falou que era bom pra gente surdo né?" (E1, M, 93 anos).

"Quem mais me falou do aparelho foi dona Lourdes, minha esposa. Ela fala assim: - Você não escuta". (E2, M, 76 anos).

"O pessoal estava cobrando muito porque eu tinha que usar o aparelho, o meu marido, filho [...] Foi pela insistência da família." (E3, F, 44 anos).

"...o pessoal aqui de casa, os familiares [...] falaram que eu não tava escutando." (E5, M, 87 anos).

"Esse povo que me põe doida, que me carrega e me levou. Eles que têm vontade. Ah! eles fala que eu tenho que usar, mas eu não quero usar." (E6, F, 82 anos).

"Ah pai o senhor está surdo" isso e aquilo, aquele negócio de filho, né?! Mas eu não queria ele porque eu não tava surdo [...] Minhas filhas que inventaram: "vamo lá fazer esses exames para o senhor usar esse aparelho" (E9, M, 88 anos).
}

Por meio das reflexões, a fenomenologia revela como as pessoas sentem, pensam, interagem no dia a dia (TERRA et al., 2006). Observou-se pelas falas a cobrança dos familiares, a negação do indivíduo quanto à surdez e a aceitação em colocar o aparelho para satisfazer os familiares.

A incapacidade auditiva pode gerar tensão no ambiente familiar. A dificuldade para ouvir provoca um incômodo na família, propiciando a intolerância para lidar com o problema levando a não ter uma interação afetiva com o indivíduo com perda auditiva (FIALHO et al., 2009; MONDELLI, MAGALHÃES, LAURIS, 2011; MONDELLI, SOUZA, 2012). Estudo realizado na Clínica de Fonoaudiologia da Faculdade de Odontologia de Bauru, Universidade de São Paulo, mostrou que a queixa dos familiares pode levar a pessoa com déficit auditivo a um quadro de frustração fazendo-a procurar por tratamento (YAMAMOTO, FERRARI, 2012).

A minoria procurou o serviço de saúde auditiva por iniciativa própria e há aquele que comentou sobre a indicação médica:

"Eu mesmo quis ir lá, foi por meu interesse mesmo. Eu queria melhorar minha saúde, né? Queria ouvir mais" (E7, F, 87 anos).

"Por conta própria, porque eu queria ter uma saúde boa, né?" (E8, F, 85 anos).

"É porque os ouvido arruinava, inflamava e rebentava, então eu ia fazer consulta [...] e o médico falou comigo que eu tinha que usar o aparei. [...]eu só fui fazê a consulta e o 
médico falou que eu tenho que colocá o aparei e fui e fiz. (E10, F, 72 anos).

\section{AS EXPECTATIVAS DOS USUÁRIOS EM RELAÇÃO AOS BENEFÍCIOS DO AASI}

Em relação às expectativas dos usuários, alguns indivíduos queriam algo específico, ou seja, uma situação que mais gostaria de ouvir.

"Ouvir a serra, tanta coisa... oração e tudo." (E1, M, 93 anos).

"Ouvir um carro ou moto, qualquer coisa." (E2, M, 76 anos).

"Eu sempre tive vontade de ouvir os sons dos passarin cantando, meu pai sempre teve criação de passarin e eu nunca escutei, aí eu pus o aparelho pra ouvir." (E3, F, 44 anos).

"Ouvir melhor, entender melhor as palavras." (E4, F, 82 anos).

Mas, houve aqueles indivíduos que construíram expectativas positivas relacionadas aos benefícios da utilização e adaptação da prótese auditiva convencional, e ficaram frustrados após o uso, conforme pode ser observado nas seguintes falas:

"Ah! eu esperava que o negócio fosse melhor [...] Que eu ia ter bom resultado. E ouvir direitinho." (E5, M, 87 anos).

"Eu esperava ouvir o que eu não tava entendendo." (E7, F, 87 anos).

O enfoque da investigação fenomenológica é o que a pessoa experimenta em relação a um fenômeno e como o interpreta. As experiências vividas dão significado à percepção de cada indivíduo sobre um determinado fenômeno, interpretando-o e descrevendo-o (TERRA et al., 2006). Portanto, aqui se preocupou em conhecer a experiência ao utilizar o AASI.

Pesquisa realizada em Curitiba - PR (GUARINELLO et al., 2013) com usuários de AASI, devido presbiacusia, revelou que antes da sua utilização os idosos poupavam de se interagir em grupos sociais na qual trazia um sentimento de tristeza e depressão e que após a colocação do aparelho houve melhora em relação a esses sentimentos. Mas, houve aqueles que apresentaram insatisfação e dificuldades, isto porque a percepção do handicap auditivo pode variar muito de indivíduo para indivíduo, dependendo do gênero, idade, fator socioeconômico e aceitação da deficiência.

As falas no presente estudo foram diferentes em relação aos sentimentos dos indivíduos participantes da pesquisa de Curitiba - PR (GUARINELLO et al., 2013), pois não revelaram tristeza ou depressão antes do uso do aparelho. Percebe-se, assim, que a perda auditiva afeta as pessoas de forma diversificada, portanto se deve ter em mente que os indivíduos podem ter uma mesma experiência, mas o conhecimento gerado a partir dela é diferenciado segundo a bagagem biográfica e reflexiva de cada um.

\section{MOTIVOS DO ABANDONO DO APARELHO}

A fenomenologia mostra que não basta conhecer a existência de algo, é necessário conhecê-lo em si mesmo, portanto, para compreender esse fenômeno é preciso buscar a 
descrição da experiência pelos sujeitos que o vivenciam. E é pela linguagem que se manifesta a essência daquilo que se sente (CARVALHO, VALLE, 2002). Isto posto, observou-se que as principais queixas para o abandono do aparelho auditivo foram 0 barulho e o incômodo quanto ao som produzido pelo aparelho.

"[...] por causa do barulho demais. [...] eu não conseguia andar com ele na rua não, de jeito nenhum. [...]Até andar na rua atrapalha, você vai andar na rua, ele vai tuctuctuc no ouvido" (E2, M, 76 anos).

"Barulho demais, me irritava demais. Barulho, muito barulho" (E3, F, 44 anos).

"O som era muito estranho, muito estranho mesmo. Eu ouvia parecendo que estava saindo pelo ouvido, a voz. Incomodava mesmo. Não aguento usar isso mais não." (E4, F, 82 anos).

"Eu sentia alto, incômodo e estalo. Eu ouvia ele gritando." (E7, F, 87 anos).

"Quando saí na rua não tava escutando nada, nem ouvia os barulhos dos carros. Eu não tava escutando nada, em vez de ouvir bem, tava tampando o ouvido." (E8, F, 85 anos).

"Mas, se uma pessoa conversava muito alto doía no ouvido, aí eu desisti." (E9, M, 88 anos).

No que refere ao barulho, todos nós seres humanos somos vulneráveis ao ruído (OLIVEIRA, LOPES, ALVES, 2010). Pesquisa realizada no norte de Minas Gerais apontou que uma grande parcela dos usuários não utilizava o AASI devido à dificuldade em adaptar com o aparelho auditivo em consequência do desconforto, ruídos, dores na orelha, dor de cabeça e prurido (BARBOSA et al., 2013). Estudo qualitativo com idosos mostrou que o ruído é uma das maiores dificuldades porque este gera desconforto (FIALHO et al., 2009). $\mathrm{O}$ desconforto sentido para os sons de alta intensidade pode fazer com que o paciente altere o volume do aparelho, alterando por sua vez o ganho auditivo, e podendo levar ao abandono do dispositivo (BRAGA, 2003).

Outros aspectos relatados para o abandono do aparelho foram a dificuldade em manuseá-lo, a não necessidade e a estética:

[...] Mas aquilo era muito miudinho atrás da orelha, eu não via, [...] dava um trabalho danado para colocar. Minha mulher que colocava para mim. [...] Quando passava um carro [...] eu queria diminuir, mas aquela rosquinha, desse tamaninho aqui, não dava. (E9, $M$, 88 anos).

"[...] minha esposa [...] fala assim: 'Você não escuta'. Mas eu escuto, sim." (E2, M, 76 anos).

"Mas eu não queria ele porque eu não tava surdo" (E9, M, 88 anos).

"Me sinto incomodado, né? Porque ele é grande. [...] principalmente pela estética, não senti confortável não. [...]Esse negócio grandão, é o tipo de aparelho que eu não vou (usar) de jeito nenhum."(E5, M, 87 anos).

"Acho que vou morrer sem o aparelho. [...] Eu não quero usar nada, é pelo meu gosto mesmo. [...]Eu gosto de ficar assim óh (mostrando a orelha), sem nada, só um brinco." (E6, F, 82 anos).

Tais relatos mostram que a adaptação do aparelho não deve limitar-se às medidas objetivas, mas, também, no julgamento do paciente quanto ao desempenho do aparelho, sua aceitação, benefício e satisfação (RODRIGUES et al., 2013). A orientação e esclarecimentos de dúvidas são importantes ao realizar a adaptação do aparelho e nos retornos de acompanhamento (FIALHO et al., 2009).

A dificuldade no manuseio também interfere no uso efetivo do AASI (BURITI, 
OLIVEIRA, 2012). Muitos usuários são idosos e apresentam comprometimento na destreza manual, dependendo, em alguns casos, da ajuda de terceiros. Por esta razão, os aparelhos retroauriculares são os mais indicados para pessoas idosas por serem maiores, e menos difíceis de manusear.

Estudo com idosos residentes no município de São Paulo - SP revelou que a principal razão atribuída para o abandono do aparelho foi o fato de não considerá-lo necessário, provavelmente o estigma quanto ao uso do AASI (CRUZ et al., 2013). Pesquisa realizada no estado de Tocantins revelou que quase a totalidade dos indivíduos pesquisados estava insatisfeita em relação à imagem pessoal com o uso do AASI retroauricular, mostrando existir preocupação com a estética (CARVALHO, 2007). Apesar da evolução dos aparelhos, no que refere à qualidade e versatilidade, a adaptação continua sendo um desafio devido às altas taxas de abandono (DANIELI, et al., 2011; VEIGA, MERLO, MENGUE, 2005).

Na pesquisa fenomenológica, uma das atitudes exigidas é verificar como os sujeitos podem agir para mudar a situação. Neste sentido, ao perguntá-los "Para o(a) senhor(a) voltar a usar o aparelho, o que teria que ser diferente, o que poderia ser feito para isso?", os entrevistados não souberam tecer críticas, apontar caminhos e soluções.

Sabe-se que algumas atitudes podem estar associadas aos resultados bemsucedidos do uso de aparelhos auditivos e são elas: a percepção da aceitação do aparelho, o benefício e a satisfação dos usuários (HOSFORD-DUNN, HUSH, 2000). Estas variáveis devem ser abordadas durante o processo de aconselhamento ao indivíduo deficiente auditivo (MONDELLI, MAGALHÃES, LAURIS, 2011).

\section{O NÃO RETORNO AO SERVIÇO}

Pôde ser observado nas falas que os indivíduos deste estudo não retornaram ao serviço, porém a maioria não comentou os motivos de não fazê-lo. Para aqueles que expressaram, as dificuldades do retorno estiveram presentes pelo fato de depender de outros para se deslocar e pelo esquecimento.

\footnotetext{
"Não cheguei ir não." (E5, M, 87 anos).

"Tenho que ir lá fazer novamente fazer os exames, né?" (E8, F, 85 anos).

"Pra ir lá tem que me levar lá, né?" (E1, M, 93 anos).

"Me deram uma folha, mas só que eu esqueci." (E10, F, 72 anos).
}

É importante ressaltar que a adaptação com AASI representa o início do processo de reabilitação auditiva promovido pelo Programa Nacional de Atenção à Saúde Auditiva (PNASA). Após a adaptação com o aparelho, é essencial que o paciente seja submetido à terapia fonoaudiológica na atenção básica para que possa "reaprender" a ouvir (BARBOSA et al., 2013). Usuários submetidos a treinamento auditivo apresentaram maior benefício com o AASI quando comparados aos que não foram submetidos ao mesmo procedimento (MEGALE, IÓRIO, SCHOCHAT, 2010). 
Pesquisa sobre indicadores de saúde auditiva em Minas Gerais mostrou que a reabilitação auditiva foi menor que o número de indivíduos que recebeu o aparelho. Atender o paciente apenas quando este recebe o AASI é insuficiente (MACIEL et al., 2013).

O Programa preconiza ainda que este paciente seja acompanhado periodicamente pelo serviço de média e alta complexidade para novas avaliações (FREITAS, COSTA, 2007). Desta forma, é necessário que os usuários retornem ao serviço para acompanhamento a fim de que tais dificuldades e desconfortos sejam melhor investigados e sanados, para que estes indivíduos possam obter um maior ganho auditivo, melhorando, consequentemente, a sua qualidade de vida.

\section{CONSIDERAÇÕES FINAIS}

O presente estudo utilizou uma abordagem que representa o fenômeno da experiência humana de acordo com a interpretação e compreensão, concedendo a observação dos respectivos dados encontrados que mostraram as dificuldades na adaptação do aparelho auditivo.

A percepção da aceitação do aparelho pelo próprio indivíduo, durante o aconselhamento, e a satisfação ou não satisfação durante o retorno deverão ser avaliadas de forma descritiva em uma conversa natural e espontânea.

Acredita-se que a assistência domiciliar aos usuários de aparelho de amplificação sonora individual poderá facilitar o uso deste. As falas apontam para a importância do fonoaudiólogo em identificar aqueles faltosos e os motivos do não retorno.

Além de respeitar as necessidades de cada sujeito, um aspecto que deve ser considerado no processo de adaptação do AASI é incluir a participação da família. Com o aconselhamento aos familiares, é possível haver maior aceitação do problema e motivação ao uso do aparelho auditivo. Afinal, eles são os incentivadores para a sua utilização.

Uma estratégia que talvez possa diminuir o abandono diz respeito à atuação dos agentes comunitários da saúde. O não retorno ao serviço poderá ser identificado pelo fonoaudiólogo e com o apoio do agente comunitário da saúde, as faltas provavelmente poderão ser minimizadas. Quando capacitados na área da audição poderão, durante as visitas domiciliares, identificar aqueles que abandonaram o AASI, observar as necessidades e dificuldades do usuário e auxiliar na adesão do paciente no programa de reabilitação, mostrando a importância dos retornos em todas as etapas do processo de adaptação.

\section{REFERÊNCIAS}

BARBOSA, M. R.; MEDEIROS, D. S.; RIBEIRO, G. M.; ROSSI-BARBOSA, L. A. R.; CALDEIRA, A. P. Satisfaction with hearing aids among users of hearing health services. 
Audiol Commun Res. São Paulo, v. 18 n. 4, p. 262-269, 2013.

BARBOSA, M. R.; MEDEIROS, D. S.; ROSSI-BARBOSA, L. A. R.; CALDEIRA, A. P. Selfreported outcomes after hearing aid fitting in Minas Gerais, Brazil. CoDAS, v. 27 n. 1, p. 21-28, 2015.

BRAGA, S. R. S. Considerações básicas sobre o processo de indicação, seleção e adaptação de próteses auditivas. In: Braga, SRS. Conhecimentos essenciais para atender bem o usuário com prótese auditiva. São José dos Campos: Pulso, 2003. p.11-15.

BRASIL. Ministério da saúde. Portaria no 587, de 08 de outubro de 2004. Institui a Política Nacional de Atenção à Saúde Auditiva. Brasília: Ministério da Saúde; 2004a.

BRASIL. Ministério da Saúde. Portaria no 2.073, de 28 de setembro de 2004. Institui a Política Nacional de Atenção à Saúde Auditiva. Brasília: Ministério da Saúde; 2004b.

BURITI, A. K. L.; OLIVEIRA, S. H. S. Hearing aid adaptation in users assisted by the Unified Health System. Soc. Bras. Fonoaudiol. São Paulo, v. 17, n. 1, p. 41-46, 2012.

CARVALHO, J. S. A. Satisfaction of elderly with hearing aid conceded in Tocantins state. Int. Otorrinolaringol. São Paulo, v.11, n. 4, p. 416-426, 2007.

CARVALHO, M. D. B.; VALLE, E. R. M. A pesquisa fenomenológica e a enfermagem. Acta Scientiarum. Health Scienc. Maringá, v.24 n. 3, p. 843-847, 2002.

COOK, J. A.; HAWKINS, D. B. Outcome measurement for patients receiving hearing aid services. Laryngoscope. v. 117, n. 4, p. 610-613, 2007.

CRUZ, M. S.; LIMA, M. C. P.; SANTOS, J. L. F.; DUARTE, Y. A. O.; LEBRÃO, M. L.; RAMOS-CERQUEIRA, A. T. A. Uso de aparelho de amplificação sonora individual por idosos: estudo SABE - saúde, bem-estar e envelhecimento. Audiol Commun Res. São Paulo, v. 18, n. 2, p. 133-142, 2013.

DANIELI, F.; CASTIQUINI, E. A. T.; ZAMBONATTO, T. C. F.; BEVILACQUA, M. C. Avaliação do nível de satisfação de usuários de aparelhos de amplificação sonora individuais dispensados pelo Sistema Único de Saúde. Soc. Bras. Fonoaudiol. São Paulo, v.16, n. 2, p. 152-159, 2011.

DIDONÉ, D. D.; VELLOZO, F. F.; GARCIA, M. V.; FEDOSSE, E. Caracterização dos candidatos ao uso de próteses auditivas em um serviço de saúde auditiva regional do estado do Rio Grande do Sul. Saúde. Santa Maria, v. 40, n. 2, p.67-72, 2014.

FIALHO, I. M.; BORTOLI, D.; MENDONÇA, G. G.; PAGNOSIM, D. F.; SCHOLZE, A. S. Percepção de idosos sobre o uso de AASI concedido pelo Sistema Único de Saúde. CEFAC. São Paulo, v. 11, n. 2, p. 338-344, 2009.

FREITAS, C. D.; COSTA, M. J. Processo de adaptação de próteses auditivas em usuários atendidos em uma instituição pública federal - parte I: resultados e implicações com o uso da amplificação. Bras. Otorrinolaringol. São Paulo, v. 73 n. 6, p. 744-751, 2007.

GUARINELLO, A. C.; MARCELOS, S. B.; RIBAS, A.; MARQUES, J. M. Análise da percepção de um grupo de idosos a respeito de seu handicap auditivo antes e após o uso 
do aparelho auditivo. Bras. Geriatr. Gerontol. Rio de Janeiro, v. 16, n. 4, p. 739-745, 2013.

HOSFORD-DUNN, H.; HUSH, J. L. Acceptance benefit and satisfaction measures of hearing aid user attitudes. In: Sandlin RE. Hearing aid amplification: Technical and clinical considerations. 2. ed. San Diego California: Singul. Publish. Group. 2000. p. 46788.

MACIEL, F. J.; JANUARIO, G. C.; HENRIQUES, C. M. A.; ESTEVES, C. C.; SILVA, M. A.; CARVALHO, S. A. S. Indicadores de Saúde Auditiva em Minas Gerais: um estudo por macrorregião. Audiol Commun Res. São Paulo, v. 18, n. 4, p. 275-284, 2013.

MARTINS, J. Um enfoque fenomenológico do currículo: a educação como poíesis. São Paulo: Cortez, 1992.

MEGALE, R. L.; IÓRIO, M. C. M.; SCHOCHAT, E. Treinamento auditivo: avaliação do benefício em idosos usuários de próteses auditivas. Pró-Fono. São Paulo, v. 22 n. 2, p. 101-106. 2010.

MINAYO, M. C. O desafio do conhecimento: pesquisa qualitativa em saúde. 9. ed. São Paulo: Hucitec; 2006.

MONDELLI, M. F. C. G.; MAGALHÃES, F. F.; LAURIS, J. R. P. Cultural adaptation of the SADL (satisfaction with amplification in daily life) questionaire for Brazilian Portuguese.

Braz. j. of otorhinolaryngol. São Paulo, v. 77, n. 5, p. 563-572, 2011.

MONDELLI, M. F. C. G.; SOUZA, P. J. S. Quality of life in elderly adults before and after hearing aid fitting. Braz. j. of otorhinolaryngol. São Paulo, v. 78, n. 3, p. 49-56, 2012.

OLIVEIRA, J. R. M.; LOPES, E. S.; ALVES, A. F. Speech perception of hearing impaired people using a hearing aid with noise supression algorithms. Braz. j. ofotorhinolaryngol. São Paulo, v. 76, n. 1, p.14-17, 2010.

PENTEADO, R. Z.; SERVILHA, E. A. M. Fonoaudiologia em saúde pública/coletiva: compreendendo prevenção e o paradigma da promoção da saúde. Distúrb. Comun. v. 16, n. 1, p.107-116. 2004.

RODRIGUES, C. C. C.; AURÉLIO, F. S.; SILVA, V. B.; LOPES, T. A. Benefit provided by the use of individual amplification device in the elderly from a hearing health program in Porto Velho-RO. CEFAC. São Paulo, v. 15, n. 5, p. 1170-1180. 2013.

TERRA, M. G.; SILVA, L. C.; CAMPONOGARA, S.; SANTOS, E. K. A.; SOUZA A. I. J.; ERDMANN, A. L. Na trilha da fenomenologia: um caminho para a pesquisa em enfermagem. Text. Context. Enferm. Florianópolis, v. 15, n. 4, p. 672-78. 2006;

TRIVIÑOS, A. N. S. Introdução à pesquisa em ciências sociais: a pesquisa qualitativa em educação. 2. ed. São Paulo: Atlas; 1994.

VEIGA, L. R.; MERLO, A. R. C.; MENGUE, S. S. Satisfaction level with hearing aid in the daily life of Army Healthcare System users. Bras. Otorrinolaringol. São Paulo, v. 71 n. 1, p. 67-73, 2005.

YAMAMOTO, C. H.; FERRARI, D. V. Relationship between hearing thresholds, handicap 
and the time taken to seek treatment for hearing loss. Soc. Bras. Fonoaudiol. São Paulo, v. 17 n. 2, p.135-41, 2012.

ZANARDI, B. M. M.; YAMADA, M. O.; BEVILACQUA, M. C. A experiência do adolescente usuário de implante coclear. Psicol. Belo Horizonte, v. 15, n. 1, p. 69-89, 2009. 